\title{
Spin-orbit entanglement in time evolution of radial wave packets in hydrogenic systems
}

\author{
Marcin Turek \\ Institute of Physics, Maria Curie-Sktodowska University, 20-031 Lublin, Poland \\ Piotr Rozmej* \\ Institute of Physics, University of Zielona Góra, 65-246 Zielona Góra, Poland
}

25th October 2018

\begin{abstract}
Time evolution of radial wave packets built from the eigenstates of Dirac equation for a hydrogenic systems is considered. Radial wave packets are constructed from the states of different $n$ quantum number and the same lowest angular momentum. In general they exhibit a kind of breathing motion with dispersion and (partial) revivals. Calculations show that for some particular preparations of the wave packet one can observe interesting effects in spin motion, coming from inherent entanglement of spin and orbital degrees of freedom. These effects manifest themselves through some oscillations in the mean values of spin operators and through changes of spatial probability density carried by upper and lower components of the wave function. It is also shown that the characteristic time scale of predicted effects (called $T_{\mathrm{ls}}$ ) is for radial wave packets much smaller than in other cases, reaching values comparable to (or even less than) the time scale for the wave packet revival.
\end{abstract}

*presenting author 


\section{Introduction}

For more than fifteen years large efforts [1-7] have been made to a detailed understanding of the quantum dynamics of wave packets in simple systems like $\mathrm{H}$ atoms, hydrogenic atoms as well as in simple molecules. These theoretical investigations resulted in a good understanding of such subtle interference effects as collapse, revivals, fractional revivals of wave packets created in a variety of systems. With development of experimental techniques allowing to tailor many different desired initial states many signatures of these phenomena have been observed. A rich survey of early studies is given in review papers [8].

Radial wave packets (RWP) can be relatively easily excited by short laser pulses [9]. Their motion, at the beginning, resembles a classical motion. During later stages of the evolution packets undergo dispersion and (partial) revivals as well as fractional revivals $[10,11]$. The idea of construction of the RWP in the paper is similar to that presented in articles cited above. However, we include in our investigation the spin degrees of freedom which motion for RWP can manifest itself much earlier than for other kinds of wave packets. The most natural framework for our considerations is using relativistic wave packets built from the solutions of Dirac equation for hydrogenic systems. Such approach was used by us in [12], where we investigated circular wave packets and in [13], where we considered elliptic wave packets, both in hydrogenic systems. Results of [12-14] show that contributions given by small components of wave functions are negligible small. Therefore for time scales of motion which are relevant one can safely use an approximation in which the time evolution is calculated with non-relativistic wave functions and relativistic energies. Such approximation is simpler for analytical presentation as well as for numerical calculations. We will use this approximation throughout the paper.

\section{Construction of wave packet}

Assume that just after creation the radial wave packet has the following form

$$
\Psi_{r}(t=0)=\sum_{n} w_{n}|n l l\rangle\left(\begin{array}{l}
a \\
b
\end{array}\right)
$$


where $|n l l\rangle$ are eigenstates of the non-relativistic hydrogenic system with low angular momentum (usually assumed as $l=1$ ) and $m=l$. The weight coefficients $w_{n}=(-1)^{n} c_{n}=$ $(-1)^{n}\left(2 \pi \sigma^{2}\right)^{-1 / 4} \exp \left[-\left(n-n_{a v}\right)^{2} / 4 \sigma^{2}\right]$ are given by a Gauss distribution with mean $n_{\text {av }}$ and dispersion $\sigma$. Distributions of that type describe population of Rydberg states excited by a short laser pulse. The phase $(-1)^{n}$ is added to obtain the initial localization of the wave packet at its external turning point. The spinor $\left(\begin{array}{l}a \\ b\end{array}\right)$ determines the initial direction of the spin. The WP (11) is an approximation of the full relativistic bispinor whose initial small components are set to zero.

\section{Time evolution}

After transformation to the basis $\left|n, l, j, m_{j}\right\rangle$ one obtains (with notation $j_{>}=l+1 / 2$ and $\left.j_{<}=l-1 / 2\right)$

$$
\left|\Psi_{r}\right\rangle=\sum_{n} w_{n}\left\{a\left|n, l, j_{>}, j_{>}\right\rangle+b\left(\sqrt{\frac{1}{2 l+1}}\left|n, l, j_{>}, j_{<}\right\rangle+\sqrt{\frac{2 l}{2 l+1}}\left|n, l, j_{<}, j_{<}\right\rangle\right)\right\} .
$$

In the basis $\left|n, l, j, m_{j}\right\rangle$ time evolution of each state is given by an exponential factor $\exp \left(-i E_{n l}^{+} t / \hbar\right)$ or $\exp \left(-i E_{n l}^{-} t / \hbar\right)$, where $E_{n l}^{+}$and $E_{n l}^{-}$are energy eigenvalues for $j_{>}=l+1 / 2$ and $j_{<}=l-1 / 2$, respectively. Precisely

$$
E_{n l}^{ \pm}=m_{0} c^{2}\left[1+\frac{(Z \alpha)^{2}}{\left[n-j_{\gtrless}-\frac{1}{2}+\sqrt{\left(j_{\gtrless}+\frac{1}{2}\right)^{2}-(Z \alpha)^{2}}\right]^{2}}\right]^{-1 / 2} .
$$

Applying time evolution in that basis and transforming back to the $\left|n, l, s, m_{s}\right\rangle$ basis one obtains the wave packet after time $t$ in the form $\left|\Psi_{r}(t)\right\rangle=\left(\begin{array}{c}\left|\Psi_{1}\right\rangle \\ \left|\Psi_{2}\right\rangle\end{array}\right)$, where the upper $\Psi_{1}(t)$ and the lower $\Psi_{2}(t)$ component of the spinor are given by

$$
\begin{aligned}
\Psi_{1}(t)=\sum_{n} w_{n} & \left\{a \exp \left(-i E_{n l}^{+} t / \hbar\right)|n l l\rangle\right. \\
& \left.+b \frac{\sqrt{2 l}}{2 l+1}\left(\exp \left(-i E_{n l}^{+} t / \hbar\right)-\exp \left(-i E_{n l}^{-} t / \hbar\right)\right)|n l l-1\rangle\right\}
\end{aligned}
$$




$$
\Psi_{2}(t)=\sum_{n} w_{n}\left\{b \frac{1}{2 l+1}\left(\exp \left(-i E_{n l}^{+} t / \hbar\right)+2 l \exp \left(-i E_{n l}^{-} t / \hbar\right)\right)|n l l\rangle\right\}
$$

Such wave packet is localized only in the radial coordinate, hence the radial density probability of the components

$$
\rho_{1}(r)=r^{2} \int d \Omega\left|\Psi_{1}(r, \theta, \phi)\right|^{2} \quad \text { and } \quad \rho_{2}(r)=r^{2} \int d \Omega\left|\Psi_{2}(r, \theta, \phi)\right|^{2}
$$

are convenient quantities illustrating the wave packet motion. The integration over angular coordinates leads to formula

$$
\begin{aligned}
\rho_{1}(r)= & r^{2}\left(a^{2}\left|\sum_{n} w_{n} R_{n}(r) \exp \left(-i E_{n l}^{+} t / \hbar\right)\right|^{2}\right. \\
& \left.+b^{2} \frac{2 l}{(2 l+1)^{2}}\left|\sum_{n} w_{n} R_{n}(r)\left(\exp \left(-i E_{n l}^{+} t / \hbar\right)-\exp \left(-i E_{n l}^{-} t / \hbar\right)\right)\right|^{2}\right) \\
\rho_{2}(r)= & r^{2}\left(b^{2} \frac{1}{(2 l+1)^{2}}\left|\sum_{n} w_{n} R_{n}(r)\left(\exp \left(-i E_{n l}^{+} t / \hbar\right)+2 l \exp \left(-i E_{n l}^{-} t / \hbar\right)\right)\right|^{2}\right),
\end{aligned}
$$

where $R_{n}(r)$ denotes the radial part of the wave function $\langle\boldsymbol{r} \mid n l m\rangle$.

Fig. 1illustrates the time evolution of the radial wave packet with $n_{\mathrm{av}}=80, a=0, b=1$, what corresponds to initial spin antiparallel to $O z$ axis. The wave packet exhibits a kind of breathing motion, moving towards the center and reassembling itself (approximately) after one classical period at the external turning point.

Periodicity of the motion is well seen with the help of the autocorrelation function $A(t)=$ $\langle\Psi(t) \mid \Psi(0)\rangle$. For radial wave packet it reads as

$$
A(t)=\sum_{n l m} w_{n}^{2}\left\{\left(a^{2}+b^{2} \frac{1}{2 l+1}\right) \exp \left(-i E_{n l}^{+} t / \hbar\right)+b^{2} \frac{2 l}{2 l+1} \exp \left(-i E_{n l}^{-} t / \hbar\right)\right\} .
$$

The plot of $|A(t)|^{2}$ for wave packets with $n_{\text {av }}=80, Z=92$ and two different values of $\sigma$ is presented in Fig. 2 ,

Even for short time evolution presented in fig. 10ne can see a transfer of the probability density from one component of the spinor to the other already after one classical period. It shows that the time scale of the period of spin-orbit motion is for RWP substantially 
smaller than for circular or elliptic WP. The period of spin-orbit motion is determined by the splitting of energy levels for which $n=n_{\mathrm{av}}$ is maximally populated in $\mathrm{WP}$ and spin projections are opposite

$$
T_{\mathrm{ls}}=\frac{2 \pi \hbar}{\left|E_{n_{a v}}^{+}-E_{n_{a v}}^{-}\right|} \simeq \frac{4 \pi n_{a v}^{3}}{Z^{4} \alpha^{2}}=\frac{2 l(l+1)}{(Z \alpha)^{2}} T_{\mathrm{cl}} .
$$

The result is obtained using lowest order approximation for relativistic energies in a hydrogenic system. It is clear that for RWP, whose $l=1, T_{\mathrm{ls}}$ can be even smaller than $T_{\text {rev }}$, particularly for large $Z$.

A hierarchy of time scales is defined as in [12]. Writing the energy as function of single quantum number $n$, for $n=n_{\mathrm{av}}$ we define a hierarchy of times

$$
\frac{1}{k !}\left(\frac{d^{k} E}{d n^{k}}\right)_{n=n_{\mathrm{av}}}=\frac{2 \pi \hbar}{T_{k}}, \quad k=1,2,3, \ldots .
$$

For $k=1$ we obtain the classical Kepler time $T_{\mathrm{cl}}$, for $k=2$ the revival time $T_{\text {rev }}$ and so on. Fig. 3 presents the three time scales $T_{\mathrm{cl}}, T_{\mathrm{rev}}$ and $T_{\mathrm{ls}}$ for RWP as functions of $n_{\mathrm{av}}$ for different $Z$. It is clear that for $Z>60 T_{\mathrm{ls}}$ becomes comparable to $T_{\mathrm{rev}}$ and even shorter for $n_{\mathrm{av}}>50$. Because lifetimes of wave packets with respect to radiative decay are about two orders of magnitude larger than $T_{\mathrm{ls}}$ (according to $[15] T_{\mathrm{ls}} / T_{\text {raddec }} \approx 0.06$ ) it is much bigger chance to observe effects of spin-orbit entanglement for RWP than for any other WP.

\section{Expectation values of spin operators}

Expectation values of spin operators can be easily obtained using equations (4). For RWP they have a simple structure

$$
\begin{aligned}
\left\langle\sigma_{x}\right\rangle_{t} & =a b \sum_{n}\left|w_{n}\right|^{2}\left[\frac{2 l}{2 l+1}+\frac{4 l}{2 l+1} \cos \left(\omega_{n} t\right)\right] \\
\left\langle\sigma_{y}\right\rangle_{t} & =a b \sum_{n}\left|w_{n}\right|^{2} \frac{4 l}{2 l+1} \sin \left(\omega_{n} t\right) \\
\left\langle\sigma_{z}\right\rangle_{t} & =\sum_{n}\left|w_{n}\right|^{2}\left[a^{2}-b^{2} \frac{(2 l+1)(2 l-1)}{(2 l+1)^{2}}-b^{2} \frac{8 l}{(2 l+1)^{2}} \cos \left(\omega_{n} t\right)\right],
\end{aligned}
$$

where $\omega_{n}=\left(E_{n l}^{+}-E_{n l}^{-} / \hbar\right)$. The terms containing $\cos \left(\omega_{n} t\right)$ and $\sin \left(\omega_{n} t\right)$ indicate that at the beginning one can expect the spin precession followed by the spin collapse implied 
by nonlinear dependence of frequencies $\omega_{n}$ on $n$. This behaviour is clearly seen in the upper part of fig. 4. where for $t / T_{\mathrm{ls}} \in(0,5)$ spin vector makes several rotations while for $t / T_{\mathrm{ls}} \in(5,20)$ stays almost constant. At that times the length of spin vector is reduced to $\boldsymbol{\sigma} \approx 0.55$ which means that the part of spin angular momentum in dynamically transferred into orbital motion. Later, for $t / T_{\mathrm{ls}} \in(20,33)$ spin revives (at half of $T_{\mathrm{ls}_{2}}=(2 / 3) n_{\mathrm{av}} T_{\mathrm{ls}}$, that is $t \approx 26.7 T_{\mathrm{ls}}$ for $\left.n_{\mathrm{av}}=80\right)$. The high peak of autocorrelation function and the larger length of spin vector presented in the lower part of fig. 4 confirm the predicted time of the spin revival.

The spin precession is accompanied by the revivals of spatial probability density. It is visible in the autocorrelation function and spin components (fig. 4) and in details in fig. [5 displaying the spatial probability density for some particular times.

The inherent entanglement of the spatial and spin degrees of freedom manifested already for short times in fig. 1 can be illustrated with the help of quantum carpet - space-time plots of the WP evolution [16]. Such space-time plot presenting time evolution of $\rho_{1}$ and $\rho_{2}$ separately is shown in fig. 6. One sees that if the initial WP has only the $\Psi_{2}$ component there is a transfer of probability density to the other component and back. This transfer is governed precisely by $T_{\mathrm{ls}}$ time scale.

\section{Conclusions}

We have discussed the time evolution of RWP in hydrogenic systems using a suitable approximation of relativistic approach. The main relativistic effect is the appearance of the new time scale due to the spin-orbit coupling. As shown above this time scale can be much smaller for the radial WP than for previously discussed cases of circular [12] or elliptic [13] WP. This fact implies that in principle experimental observations of some spin-orbit effects may become possible with existing techniques.

\section{Acknowledgement}

P. Rozmej thanks for support by the Polish Ministry of Scientific Research and Information Technology under the (solicited) grant No PBZ-MIN-008/P03/2003. 


\section{References}

[1] J. Parker and C.R. Stroud Jr., Phys. Rev. Lett. 56716 (1986).

[2] I.Sh. Averbukh and N.F. Perelman, Phys. Lett. A139 449 (1989); Zh. Eksp. Teor. Fiz. 96 818 (1989); Sov. Phys. JETP 69464 (1989); Usp. Fiz. Nauk 16141 (1991); Sov. Phys. Usp. 34572 (1991).

[3] N. Nauenberg, J. Phys. B: At. Mol. Opt. Phys. 23 L385 (1990).

[4] Z. Dačic-Gaeta and C.R. Stroud Jr., Phys. Rev. A42 6803 (1990).

[5] A. Peres, Phys. Rev. 475196 (1993).

[6] R. Bluhm and V.A. Kostelecky, Phys. Rev. A 50 R4445 (1994); Phys. Lett. A 200308 (1995); Phys. Rev. A 514767 (1995).

[7] R. Bluhm, V.A. Kostelecky and J.A. Porter, Am. J. Phys. 64944 (1996).

[8] G. Alber and P. Zoller, Phys. Reports 5231 (1991);

B.M. Garraway and K.A. Suominen, Rep. Prog. Phys. 58365 (1995).

[9] G. Alber, H. Ritsch, and P. Zoller. Phys. Rev. A 341058 (1986).

[10] J.A. Yeazell, M. Mallalieu, J. Parker, and C.R. Stroud Jr. Phys. Rev. A 405040 (1989).

[11] J.A. Yeazell, M. Mallalieu, and C.R. Stroud Jr. Phys. Rev. Lett. 642007 (1990).

[12] R. Arvieu, P. Rozmej, and M. Turek. Phys. Rev. A 62022514 (2000).

[13] P. Rozmej, M. Turek, R. Arvieu, and I.Sh. Averbukh. J. Phys. A: Math. Gen. 357803 (2002).

[14] M. Turek, Relativistic effects in time evolution of wave packets, $\mathrm{PhD}$ thesis (in Polish), University Maria Curie-Skłodowska, Lublin 2002.

[15] C.E. Chang Phys. Rev. A 31495 (1985).

[16] F. Grossman, J.-M. Rost and W.P. Schleich, J. Phys. A 30, L227 (1997); P. Rozmej and R. Arvieu, Eur. Phys. J. A 5357 (1999); R. Bonifacio, I. Marzoli and W.P. Schleich, J. Mod. Optics 472891 (2000). 


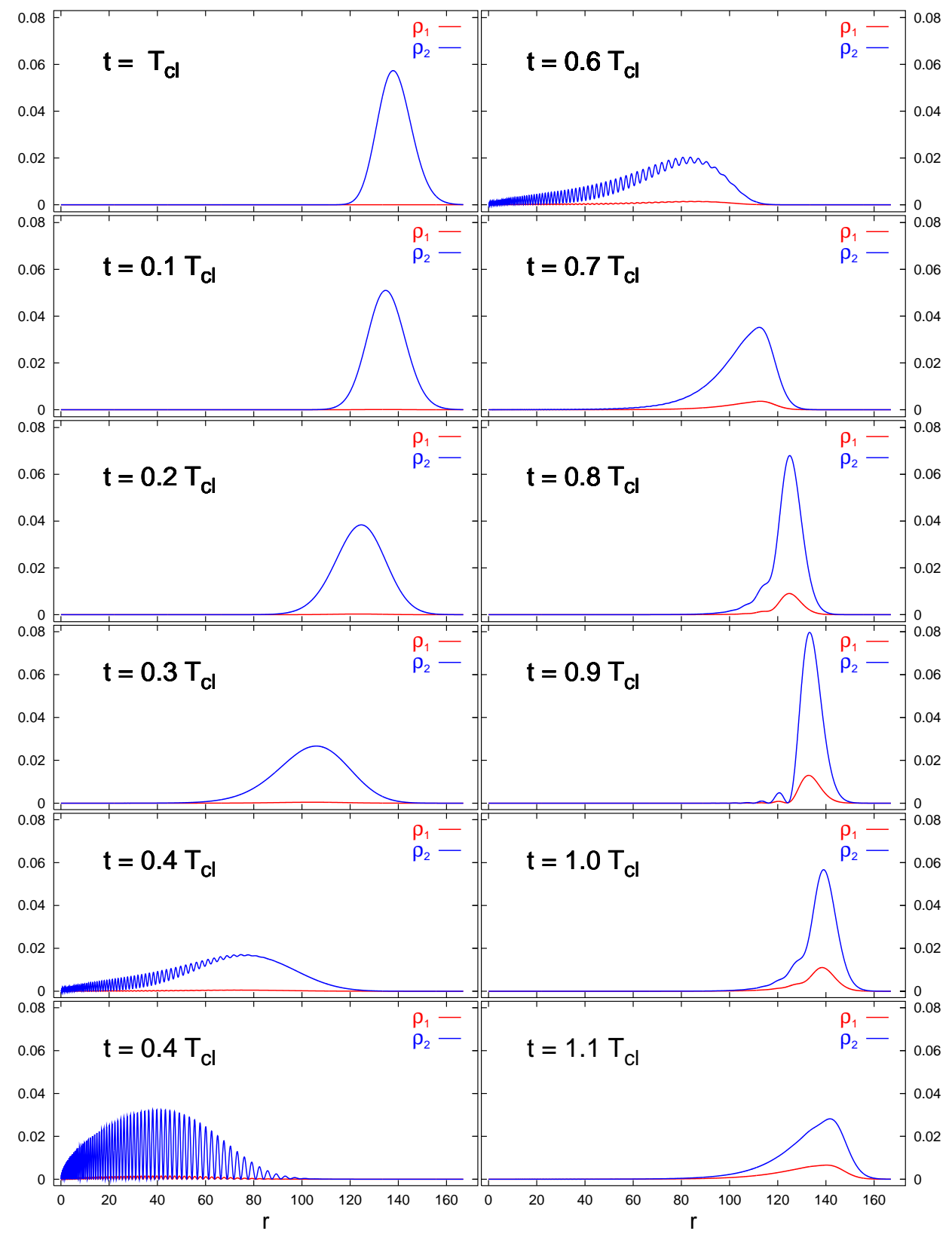

Figure 1: Short time scale evolution of the radial wave packet with $n_{\mathrm{av}}=80, a=0, b=1$ and $\sigma=2$. 


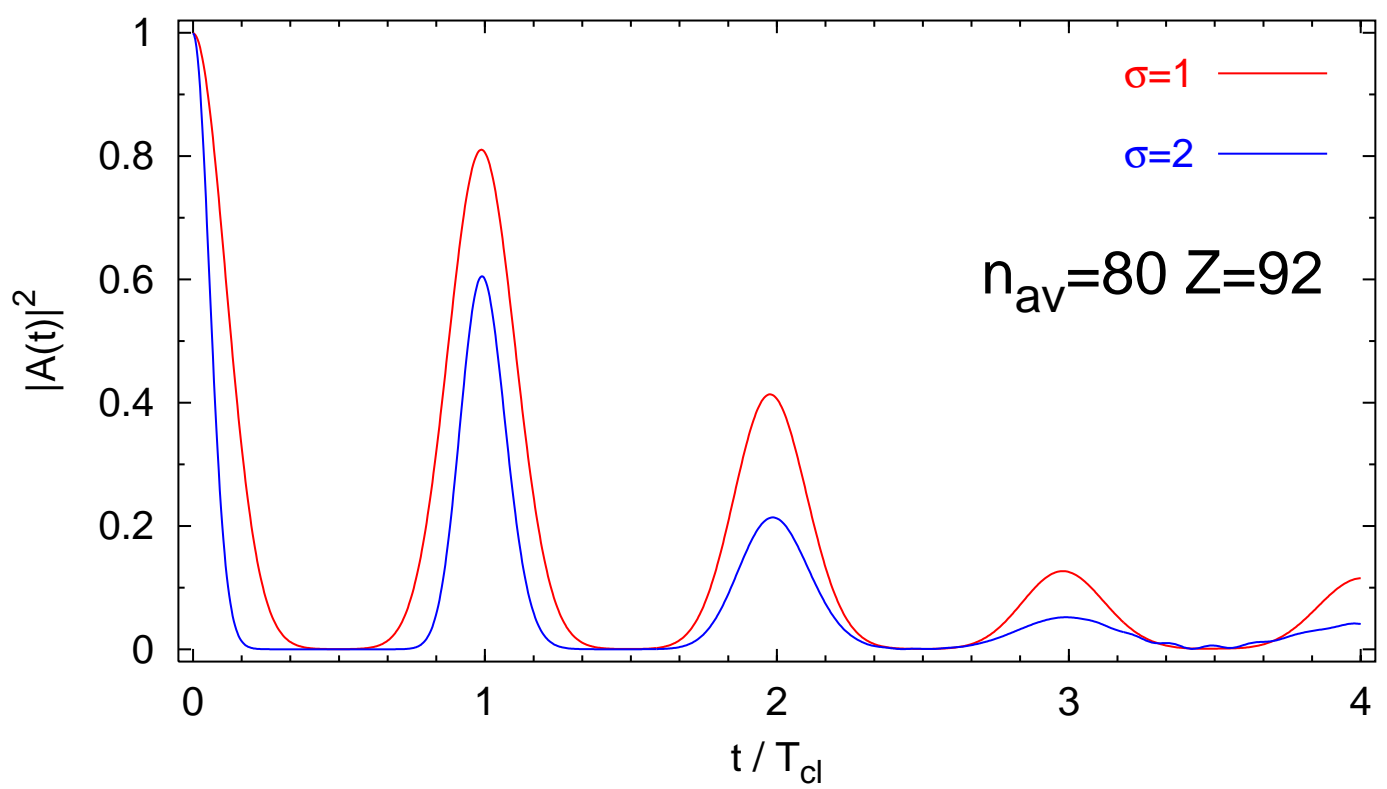

Figure 2: Autocorrelation function (squared) for radial wave packets with $n_{a v}=80, Z=92$, $\sigma=1$ and 2 . 

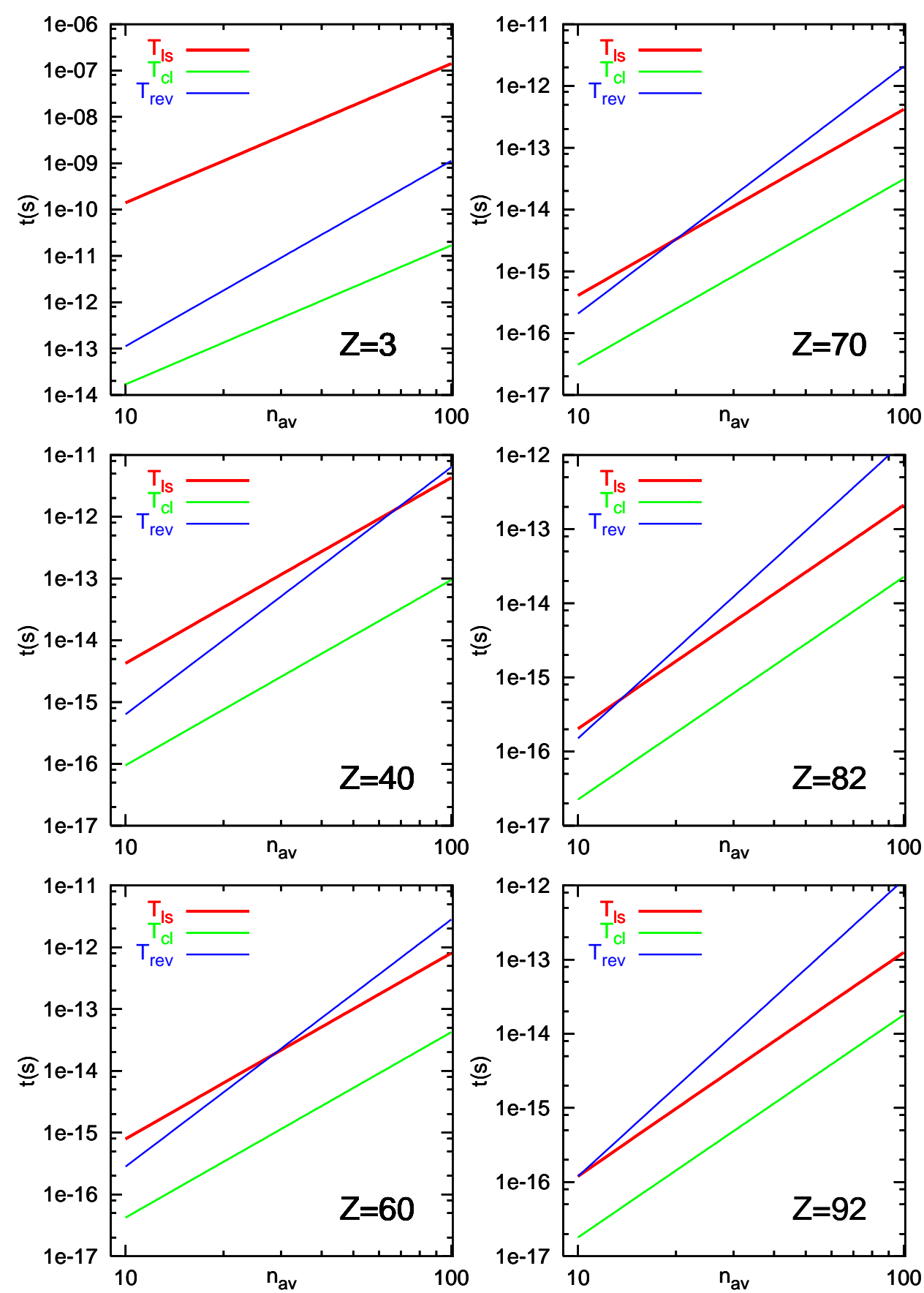

Figure 3: Log-log plot of time scales (in seconds) $T_{\mathrm{cl}}, T_{\mathrm{rev}}$ and $T_{\mathrm{ls}}$ of radial WP as function of $n_{\text {av }}$ for different $Z$. 


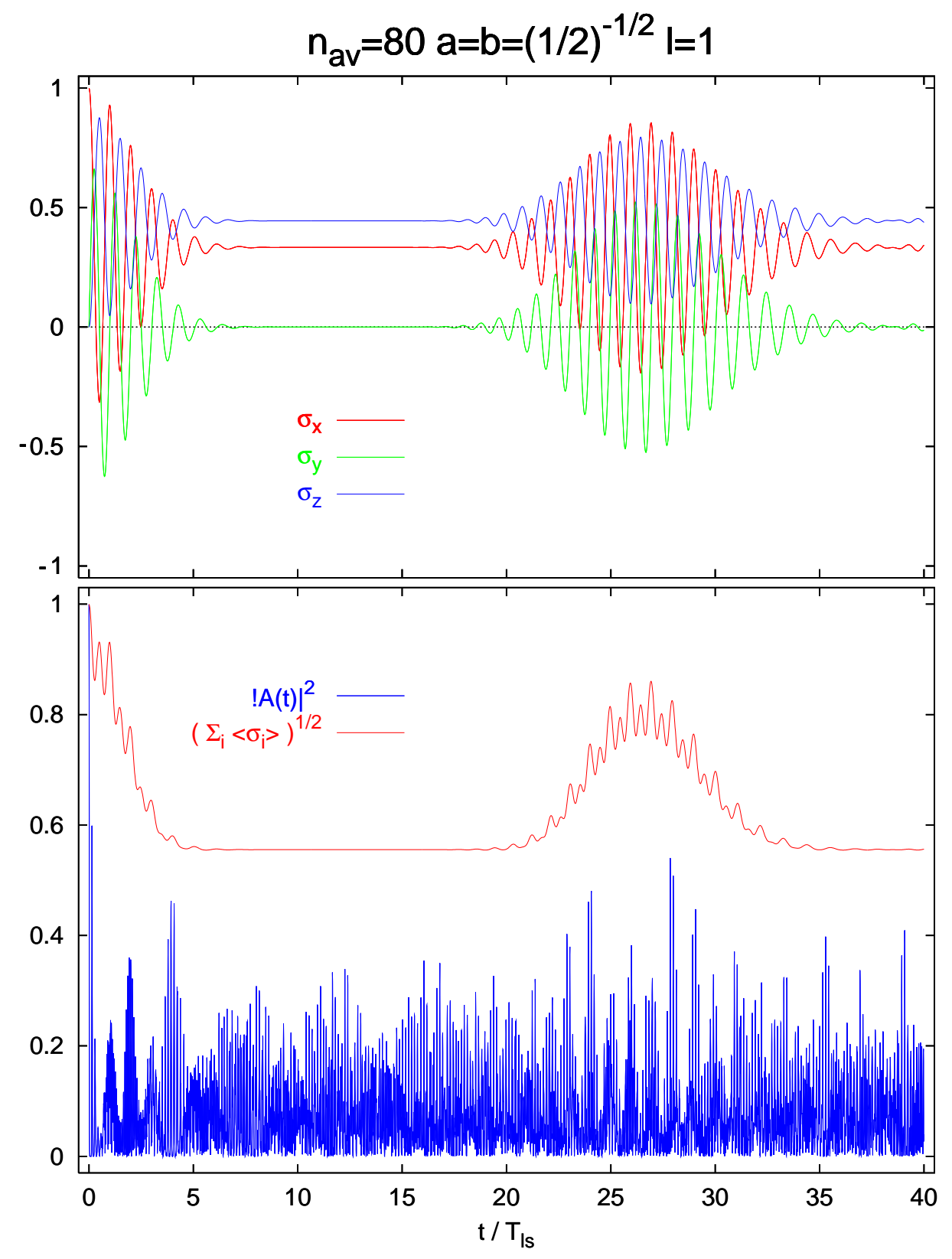

Figure 4: Time evolution of spin expectation values for RWP with $n_{a v}=80, l=1$ and $a=b$ (upper part) and the square of the autocorrelation function and the 'length' of the spin vector (lower part). 


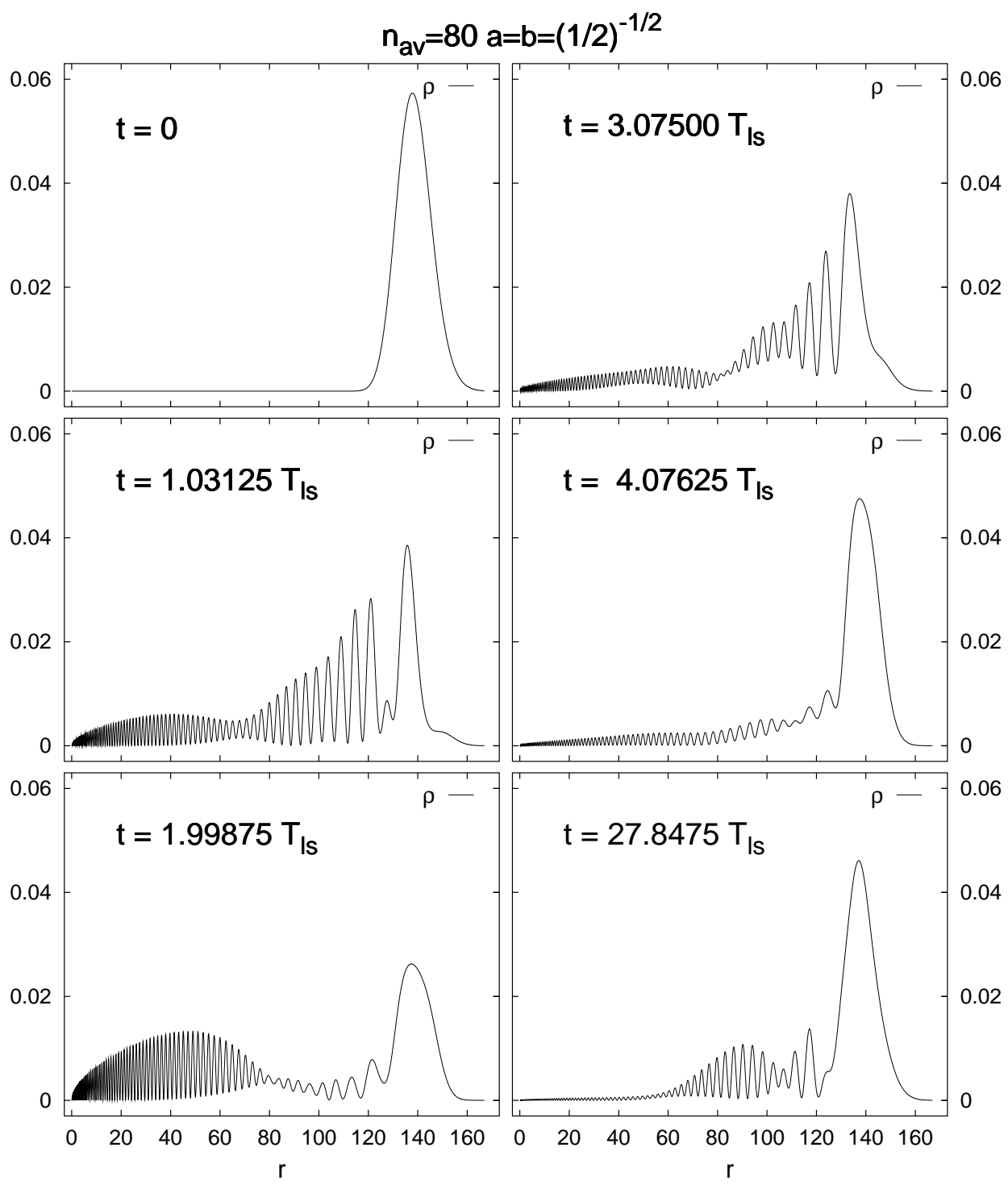

Figure 5: Radial probability density $\rho=\rho_{1}+\rho_{2}$ for RWP with $n_{\text {av }}=80, l=1$ and $a=b$ for several time instants corresponding to big values of autocorrelation function (revivals).

Figure 6: Time evolution of radial probability densities $\rho_{1}$ and $\rho_{2}$ for RWP with $n_{\mathrm{av}}=80$, $l=1, a=0, b=1$. 
This figure "cpn80.b.ls1_a1.jpg" is available in "jpg" format from: http://arxiv.org/ps/quant-ph/0404084v1 